\section{SIMULATED DESPONDENCY FOR ROBOTS IN DISTRESS}

Vicky Isley ${ }^{1}$ and Paul Smith ${ }^{2}$, National Centre for Computer Animation, Bournemouth University, Poole, BH12 5BB, U.K. Email: info@boredomresearch.net. ORCID: 10000-0003-31795304; ${ }^{20000-0001-6976-3733 . ~}$

See www.mitpressjournals.org/toc/leon/52/1 for supplemental files associated with this issue.

Submitted: 7 March 2017

\section{Abstract}

It is widely accepted that increased human interaction with natural systems is responsible for complex environmental issues, with most current thinking centered on the provision of advanced technological solutions. One response emerging from current bioinspired robotics research proposes artificial neural networks (ANNs) enhanced with artificial hormones for increased performance and efficiency. Here the authors discuss their artistic project concept, developed in collaboration with a bioinspired artificial life lab, considering the affordance of emotional robotics to develop despondency in the field.

\section{An Artistic Response to Bioinspired Robotics}

For the last two decades our artistic practice has explored an understanding of the natural world through the medium of computational technologies. When we attended the Future Emerging Art and Technology (FEAT) matchmaking event [1], we were intuitively drawn to the research behind the subCULTron project (Submarine Cultures Perform Long-Term Robotic Exploration of Unconventional Environmental Niches), which is heavily influenced by biological processes and mechanisms. subCULTron is a Future and Emerging Technologies (FET) project [2], consisting of a consortium of scientific research labs in Europe working across advanced robotics and bioinspired simulation, with the aim to achieve long-term autonomy in a learning, self-sustaining underwater culture of robots in a high-impact environment. During our FEAT residency in 2016 we immersed ourselves in subCULTron's Artificial Life Laboratory of the Karl-FranzensUniversität-Graz in Austria. This took us into a new area of consideration as we explored the transition from simulated software worlds to robotic interventions in natural environments. At the start of the residency, Daniel Moser, a researcher in the Graz AL lab, presented a simulation of the Braitenberg vehicle model, consisting of two wheels, two sensors and two artificial synapses. The simulation presented a modification with an addition of artificial hormones. This model of emotional robotics evidenced increased efficiency when faced with the task of seeking and consuming resources: "After 10,000 time-steps the agents with a hormonal system had evolved a controller that is significantly more efficient than agents without a hormonal system" [3]. Efficiencies like those evidenced in this paper can have significant value in the field. For us this did, however, raise some questions, especially when viewing the frenetic behavior of agents hungrily consuming resources with seemingly insatiable appetites. This led us to consider whether there might be an alternative vision for emotional robotics.

\section{Environmental Crisis and Melancholy}

Following Marvin Minsky's argument that "the question is not whether intelligent machines can have any emotions, but whether machines can be intelligent without any emotions" [4], it is worthy to consider the significance of negative emotions in the human condition. Current frames of reference, like those informing the Beck Hopelessness Scale [5], evaluate emotional responses to failure, including despondency, as harmful: "They interfere with normal cognitive processing, leading to impairments in perception, memory and problem solving" [6]. Clinical practice offers by way of remedy a range of therapeutic solutions from psychoactive drugs to psychotherapy.

In contrast, much commentary on twentieth-century art celebrates artifacts produced subject to a heightened negative emotional state. For example, Vincent van Gogh's archetypal anguish, present in his letter to Theo van Gogh "one feels as if one were lying bound hand and foot at the bottom of a deep, dark well, utterly helpless" [7], presents a contradiction between the adverse effects of melancholy and valued artistic expression thereof. Although we share objections to a myth of tortured genius, we do acknowledge a connection between concepts of value and negative emotion. We also argue that this specific type of value goes beyond that which satisfies standard economic interest and may, in contrast to a onedimensional pursuit of happiness, constitute a significant function in addressing complex environmental relationships. Discussing embodied cognition, Tom Ziemke and Robert Lowe reference Jesse J. Prinz's observation that "Emotions track bodily states that reliably co-occur with important organismenvironment relations, so emotions reliably co-occur with important organism-environment relations" [8]. In Against Happiness: In Praise of Melancholy, Eric G. Wilson adds "positive psychology" to a list of concerns including nuclear proliferation, global warming and environmental crisis, arguing that a current happy-centric bias tricks us to behave "as predictably and artificially as robots," warning of "wastelands of mechanistic behavior" [9].

We argue that the role of negative emotions in humans remains unclear and therefore should not be excluded from consideration in advanced robotics. It is clear that negative emotions are central to the human condition and may be important in our evaluation of complex long-term environmental challenges. Furthering the use of simulated hormones in ANNs to increase efficiency, we value a wider range of behaviors, including those that may fail a given task. In doing so, we propose that current paradigms aiming to address significant environmental concerns with technological solutions must recognize the reality of failure. Only then can they be undertaken with a genuine equitable intent.

\section{Self-Destructive Robots}

subCULTron's ambitious aim to establish a culture of robots in the Venice Lagoon, where they will collect valuable scientific data, faces the challenges of a harsh human-polluted environment. These conditions encourage a strong leaning toward highly engineered and robust solutions.

Our time in residence at the AL lab in Graz revealed that the process by which these solutions are achieved sometimes involves quickly produced test robots made from easily sourced materials, including jam jars, plastic bottles and other household waste. Mirroring this process, we constructed our own robots from plastic waste (Fig. 1), incorporating plastic bags in their construction - giving them a strong biological appearance and bringing to mind the accidental consumption of plastic, which turtles mistake for a valuable food source. This consumption comports with the findings of a recent study of seabirds predicting that "plastic ingestion is increasing in seabirds," and "that it will reach $99 \%$ of all species by 2050 " [10]. Ironically, in the lab, our robots began a process of selfconsumption, with their propellers drawing their tendrils toward themselves such that they became entangled, strangling 


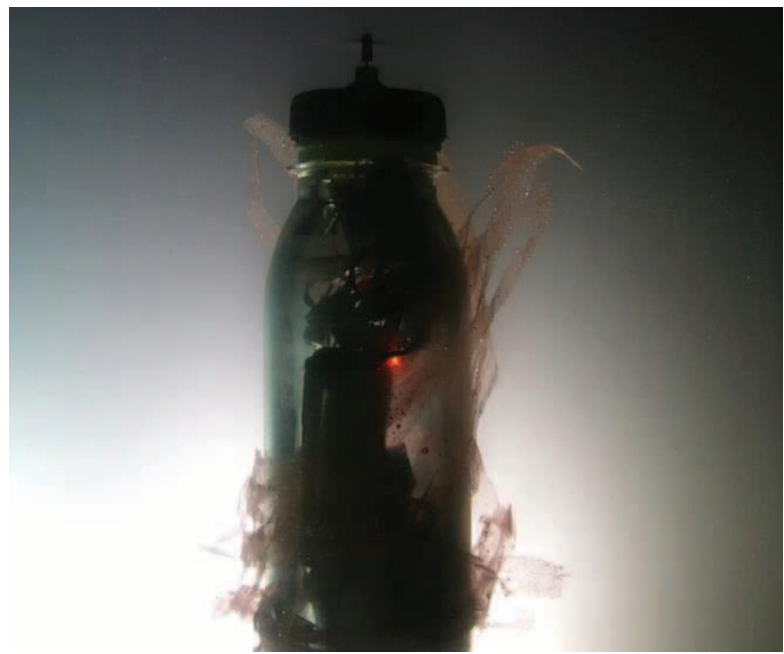

Fig. 1. boredomresearch underwater plastic waste robot. (C) boredomresearch. Photo: boredomresearch.)

themselves to death. At the time our scientific host considered this an engineering problem for which a solution could be found. It became difficult to communicate our belief that it was in itself an insightful expression of a current environmental situation, where technological solutions provide their own problems. In building robots from plastic waste we discovered a synergy between the problems of human consumption that pollute the environment and the process by which we attempt to provide solutions. This led us to reflect on and evaluate the previously discussed models of artificial hormones and the concept of emotional robotics. With current consideration centered on a use supporting an engineering paradigm we now aim to offer a fuller consideration including the more human capacity for failure.

\section{Simulating Despondency in Robotics}

Modifying and extending the Braitenberg vehicle model with simulated hormones as discussed above, we are now working on a simulation that is inspired by the idea of a culture of marine robots with limited motion capability and a narrow channel for intergroup communication. The simulated robot agents will be faced with a task of navigating their environment, conforming to a similar challenge faced by subCULTron's proposed robot ecology. In essence the agents have two inputs and two outputs connected by an artificial neural network augmented by a simulated hormone gland (Fig. 2). Each agent has the ability to propel itself vertically in a simulated liquid body becoming subject to a simulated current. It can also broadcast its emotional state. Input consists of receiving stimuli from other agents as well as from their environment when in contact with the ground. The simulation aims to explore the potential for individual agents to exhibit behavior consistent with an awareness of failure, which will occur when energy levels result in the loss of agency. It is currently unclear if recognition of a failure and subsequent expression of despondency can emerge as a natural product of the system or if it will need to be "engineered" as a solution to acknowledge and incorporate the potential for failure. Ultimately, we aim to introduce a consideration in emotional robotics that respects the fragility of a natural environment subject to high levels of human intervention at increasingly complex levels of sophistication.

What we provide may offer little within a current paradigm centered on increasingly complex technological solutions for increasingly complex problems. It is, however, consistent with a cultural perspective that precedes the strict constraints of scientific method. It forms part of a continued interaction with science where, in this case, we extend a warm hand from an artistic perspective comfortable with melancholy. In doing so we acknowledge the very human capacity for failure and despondency, for the purpose of fostering an enhanced sensibility for environmental fragility.

\section{Acknowledgments}

boredomresearch would especially like to thank Thomas Schmickl, SubCULTron project coordinator; Ronald Thenius; Joshua Cherian Varughese and Daniel Moser.

\section{References and Notes}

FEAT is an initiative of eutema GmbH (AT), Stichting Waag Society (NL) and youris.com (BE). It has received funding from the European Union's Horizon 2020 research and innovation program under grant agreement No. 686527 (H2020-FETOPEN-2015-CSA).

1. Future Emerging Art and Technology Workshop, Waag Society, Amsterdam (March 2016): www.featart.eu, accessed 14 February 2017.

2. subCULTron (2015-2019): www.subcultron.eu, accessed 27 February 2017.

3. D. Moser, R. Thenius and T. Schmickl, "First Investigations into Artificial Emotions in Cognitive Robotics," subCULTron, Artificial Life Laboratory, Karl-Franzens-Universität-Graz (Austria: 2016) p. 12:

http://zool33.uni-graz.at/artlife/sites/default/files/Paper_MESROB_2016Investigations_Artific_Emo.pdf.

4. M. Minsky, The Society of Mind (New York: Simon \& Schuster, 1986) p.163.

5. A.T. Beck, Beck Hopelessness Scale (San Antonio, TX: The Psychological Corporation, 1988).

6. S.A. McLeod, "Psychological Theories of Depression," SimplyPsychology (2015): www.simplypsychology.org/depression.html.

7. Van Gogh's Letters, "Letter from van Gogh to Theo van Gogh, The Hague, 26 January 1882": www.webexhibits.org/vangogh/letter/11/173.htm, accessed 1 October 2018 .

8. T. Ziemke and R. Lowe, "On the Role of Emotion in Embodied Cognitive Architectures: From Organisms to Robots," Cognitive Computation 1, No. 1, 104-117 (2009) p.107.

9. E.G. Wilson, Against Happiness: In Praise of Melancholy (New York: Farrar, Straus and Giroux, 2008) pp.5-9.

10. C. Wilcox, E.V. Sebille and B.D. Hardesty, "Threat of Plastic Pollution to Seabirds Is Global, Pervasive, and Increasing," Proceedings of the National Academy of Sciences of the United States of America 112, No. 38 (22 September 2015) p. 11899.

Fig. 2. Depiction of a robot agent showing arrangement of network with hormone gland. (C) boredomresearch)

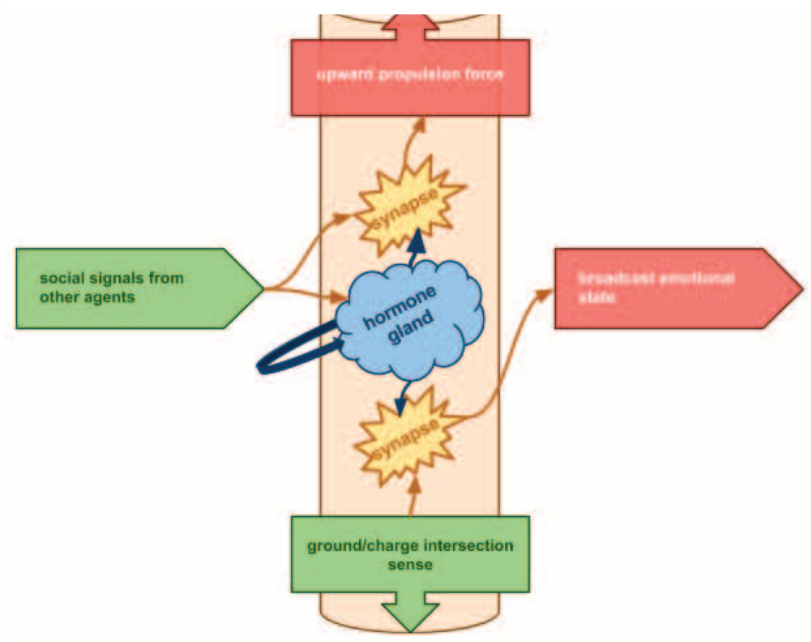

